\title{
Non-invasive Measurement of Hemoglobin for Rural India using Artificial Intelligence
}

Shoban Mude ( $\nabla$ shoban.mude@gmail.com )

Osmania University University College of Engineering https://orcid.org/0000-0002-5830-9049

\section{Research Article}

Keywords: ECG, PPG, Bluetooth, Blood Pressure (BP), SpO2, and Hemoglobin

Posted Date: February 18th, 2022

DOI: https://doi.org/10.21203/rs.3.rs-1282743/v1

License: (a) (i) This work is licensed under a Creative Commons Attribution 4.0 International License. Read Full License 


\title{
Non-invasive Measurement of Hemoglobin for Rural India using Artificial Intelligence Algorithms
}

\author{
Bhukya Rajendra Naik, Senior Member IEEE, Shoban Mude, Ph.D, and Dharavath Vennela, Research \\ Scholar
}

\begin{abstract}
The body sensor networks includes the nodes of ECG and PPG sensors, with the aim to investigate a novel technique for cuffless Blood Pressure estimation with better exactness. The sensor networks performance in the BSN, correlates various PTTs determined from ECG and PPG, with the reference $B P$ recorded at various postures. The outcome shows that SBP and DBP have better connection with PTTs determined ECG to PPG sensors. The interesting thing is values of PTT acquired from ECG to PPG sensor at fingertip are better when compared with regular methods. Later this circuit is embedded with Bluetooth module and monitors fully automatic and continuous measurement Blood Pressure, SpO2 and Hemoglobin.
\end{abstract}

Index Terms-ECG, PPG, Bluetooth, Blood Pressure (BP), SpO2, and Hemoglobin.

\section{INTRODUCTION}

Hemoglobin $(\mathrm{Hb})$ measuring methods were created over a century ago, making haemoglobin one of the earliest diagnostic bloods tests available to doctors in the early twentieth century, when laboratory medicines was still in its infancy. It is now the most commonly requested blood test, and it is conducted not just in hospital laboratories, but also in a variety of healthcare's settings, by a variety of healthcare's staff, and with a variety of sophisticated technologies. Blood vessels contain a protein called hemoglobin, which is present

This paragraph of the first footnote will contain the date on which you submitted your paper for review. It will also contain support information, including sponsor and financial support acknowledgment. For example, "This work was supported in part by the U.S. Department of Commerce under Grant BS123456."

The next few paragraphs should contain the authors' current affiliations, including current address and e-mail. For example, F. A. Author is with the National Institute of Standards and Technology, Boulder, CO 80305 USA (email: author@ boulder.nist.gov).

S. B. Author, Jr., was with Rice University, Houston, TX 77005 USA. He is now with the Department of Physics, Colorado State University, Fort Collins, CO 80523 USA (e-mail: author@lamar.colostate.edu).

T. C. Author is with the Electrical Engineering Department, University of Colorado, Boulder, CO 80309 USA, on leave from the National Research Institute for Metals, Tsukuba, Japan (e-mail: author@ nrim.go.jp). in erythrocytes (red blood cells). Around 280 million haemoglobin molecules are found in each of the $5 * 10^{\wedge 10}$ erythrocytes found in $1 \mathrm{~mL}$ of blood. Hemoglobin $(\mathrm{Hb})$ is a spherical molecule made up of two pairs of different subunits. Each component is made up of a folded polypeptide chain (globin) with a heme group (produced from porphyrin) connected to it. A single atom of iron in the ferrous (Fe 2+) state is at the core of each heme group. As a result, heme is a metalloporphyrin, which is also responsible for blood's red hue.

The primary purpose of testing ctHb is to diagnose anaemia and quantify its severity. Anemia is described as a decrease in blood oxygen carrying capacity caused by a decrease in erythrocyte counts and/or a decrease in ctHb, resulting in anaemia. If ctHb is below the reference (normal) range's lower limit. The anaemia is more severe when the ctHb is low. People and other vertebrates transport oxygen to and carbon dioxide from their bodies through blood, a crimson liquid that flows within their arteries and veins. Hemoglobin, a protein important for delivering oxygen in vertebrates' blood, is found in blood. Four subunits of its molecule are composed of iron atoms attached to haem groups.

Red blood cells make up haemoglobin. Many invertebrates, fungi, and plants contain haemoglobin and hemoglobin-like molecules. Hemoglobins may transport and control a variety of tiny molecules and ions, including carbon dioxide, nitric oxide, hydrogen sulphide, and sulphide. Haemoglobinemia is a medical disorder in which the bloods plasma contains too much haemoglobin. Haemoglobin separates from red blood cells as a side effect of intravascular hemolysis, a type of anaemia. Johann Friedrich Engelhart observed in 1825 that the ratio of iron to protein in the hemoglobins of many species is equal, and he computed the molecular mass of haemoglobin to be $n * 16000$ using the known atomic mass of iron. Oxyhemoglobin and deoxy-hemoglobin are two types of haemoglobin. Hemoglobin may be calculated in two ways: invasive and non-invasive. The blood pressure monitor, also known as a manual sphygmomanometer, has been the 
favoured method of checking a person's vitals for nearly 150 years. After an accident, one of the most common laboratory procedures is haemoglobin testing. Non-invasive haemoglobin monitoring is a relatively recent addition to the increasing list of point-of-care testing capabilities that allows for continuous, accurate, and non-invasive haemoglobin concentration monitoring.

Invasive measuring, while precise, is time-consuming, expensives, and uncomfortable for the patient, as well as posing a danger of biohazard exposure. Spectrophotometry is used in the majority of non-invasive haemoglobin measuring equipment. The Radical7 is a Pulse CO-oximeter created by Masimo Corporations (Irvine, CA) that can monitor haemoglobin concentration utilising a noninvasive, multiwavelength sensor for spot check and continuous measurement. It uses the absorption of light by haemoglobin to calculate haemoglobin concentrations by generating a number of wavelengths of light. A fingertip probe, similar to the sensor on a typical pulse oximeter, measures haemoglobin noninvasively.

Continuous haemoglobin monitors and spot check haemoglobin monitors are the two categories of haemoglobin measurement equipment. The continuous non-invasive haemoglobin monitors, as the name implies, continually measures serum haemoglobin levels and delivers real-time haemoglobin readings, much like a pulses oximeter does. The blood haemoglobin monitors are continually monitoring devices, while the spot test haemoglobin monitors are pointof-care devices. The spot check haemoglobin monitor automatically calculates blood haemoglobin levels within 1 minute of insertion. The accuracy and dependability of both of these monitors are variable, and they have yet to be clinically tested in trauma.

525 trauma patients were enrolled in the biggest prospective research on trauma patients, which comprised 1350 spot check haemoglobin readings. The automated recalibration procedure is known to affect the accuracy of volume clamp systems. Arterial blood pressure (ABP) is a critical clinical metric, and there have been various attempts to get continuous noninvasive ABP monitoring. PPG has been used to monitor blood pressure, not as a stand-alone approach based on its waveform features, but as a tool for detecting blood volume changes caused by other devices in certain circumstances.

\section{PROPOSED DESIGN}

Based on a multispectral measuring approach, a newly designed sensor gadget enables for non-invasive continuous detection of haemoglobin concentrations, oxygen saturation, and pulses. The flesh of the finger is therefore transilluminated by monochromatic light generated by LEDs with wavelengths ranging from $600 \mathrm{~nm}$ to $1400 \mathrm{~nm}$. Due to an enlarged diameter of the arteries during the systolic phase of the heart, the arteries hold more blood than during the diastolic phase. This impact only happens in arteries, not veins. Because the quantity of haemoglobin (absorber) is larger during systole and the light goes via a longer optical paths length $d$ in the arteries, the absorbance of light in tissues with arteries rises. These fluctuations in intensity are known as PPG-waves.

\section{A. Block Diagram}

The time-varying part distinguishes between venous blood absorbance (DC portion) and pulsatile absorbance (total absorbance) (AC part). A detailed model of light absorption in tissue is shown in Figure 1 for the depth at which arterial blood can be found. Photo diodes detect the transmitted light after it interacts with the tissue in a non-invasive way.

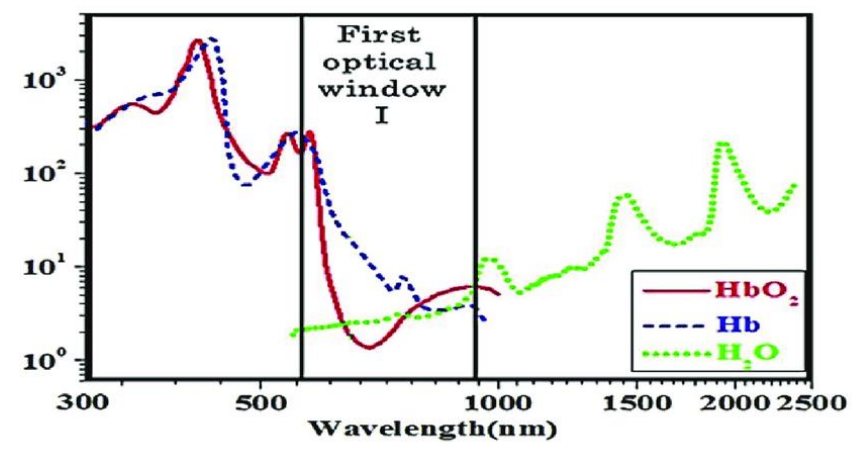

Fig.1 Absorption Spectra of Hemoglobin and Water

To measure $\mathrm{SpO} 2$ and analyze the change in haemoglobin concentration, appropriate wavelengths were used. The absorption of haemoglobin should not be affected by oxygen saturation while measuring it. That is, the measurement can only be done at so-called isobestic locations, when the extinction coefficients of $\mathrm{HHb}$ and $\mathrm{HbO} 2$ are the same. Around the wavelength of $810 \mathrm{~nm}$, such a point is known to exist. In view of the fact that the absorption coefficients of blood is identical to a solution containing hemoglobin $(\mathrm{HHb})$, hemoglobin oxygen $(\mathrm{HbO} 2)$, and water $(\mathrm{H} 2 \mathrm{O})$, it is essential to select a wavelengths value above the diagnostic window that is comparable to this absorption coefficient.

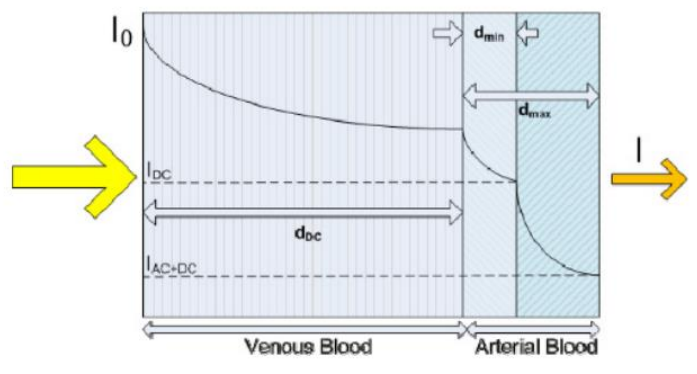

Fig.2 Model of Tissue Layer

Finally, the concentration of haemoglobin will be determined using the wavelength $\lambda_{1}=810 \mathrm{~nm}$ and $\lambda_{2}=1300 \mathrm{~nm}$. The quotient $\mathrm{H}$ (equations 1) is obtained by multiplying the AC/DC values 

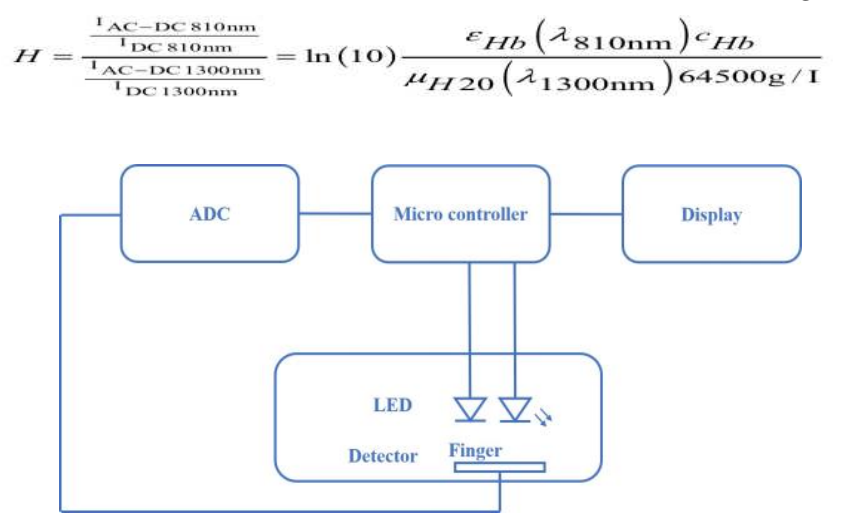

Fig.3 Block diagram of non-invasive method

\section{B. Multi-chip LEDs}

The phosphor layer on a multi-chip white LED model, as well as the encapsulating lens, should be able to track a multitude of rays from each chip. On the other hand, modeling the light pattern involves an enormous amount of computation. This method was used to create a single-chip white LED simulation. Although effective for modelling the colour angular distribution, this technique indicated that for the stimulation of the radiation pattern, there is little difference between employing a complicated phosphor model or a basic Lambertian model. If one models a single-chip white LED using a Monte Carlo simulation, then the top surface of the phosphor layer is typically considered to be the beginning Lambertian light source.

The typical Lambertian model, on the other hand, does not adequately represent the experimental findings for a phosphorcoated multi-chip LED. Figure 4 shows the problem for an 8 chip LED with the following dimensions: chip side 1 a.u., The disc diameter is 6.5 a.u., chip spacing is 0.5 a.u., and the cap has an 8 a.u. radius and a 4.2 a.u. height at the tip. It's worth noting that the traditional technique disagrees with the off-axis data. The energy distributions over the phosphor surfaces, which is dependent on geometric parameters, phosphor layer thickness, phosphor particle diameter, phosphor absorption, and other factors, is the key concern lacking in this problem, as we will discuss in the following section.
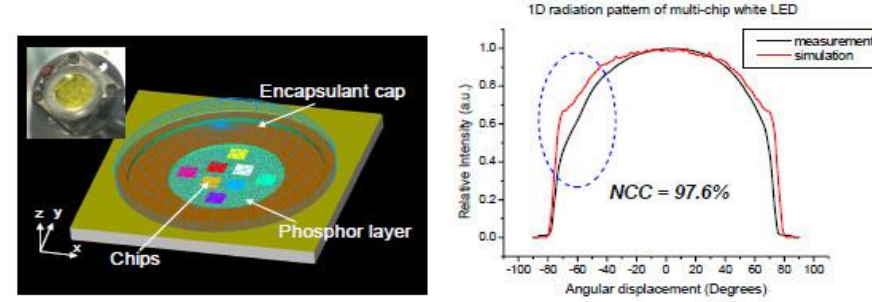

Fig.4 Multi-Chip LED

\section{OPT101 Photodiode}

The integrated photodiode OPT101 (Figure.3.5) is a type of integrated photodiodes with a built-in transimpedance amplifier on the chip. The amplifier is suitable for a batteryincluded instrument and is intended for single or dual powerserved operation. Current leakages, noise, and stray capacitances-induced gain peaking may all be reduced by combining a photodiode and a trans-impedance amplifier on a single chip. In addition, the $8.1 \times 10^{-3}$ inch 2 photodiodes has a good linearity and a small dark current in photoconductive mode. The OPT101's operating voltage spans from 2.7 to 36 volts, and its quiescent current is just 120microamps. The bandwidth of the OPT101 circuitry design is $2.5 \mathrm{kHz}$ with an external $5 \mathrm{M}$ feedback resistor and an external $10 \mathrm{pF}$ capacitor. $6106 \mathrm{~V} / \mathrm{A}$ and $5 \mathrm{~V}$ are the DC gain and actual operating voltage, respectively. As shown in Figure.2.3.2, the responsivity of OPT101 at the specified wavelengths $(735 \mathrm{~nm}$, $805 \mathrm{~nm}$, and $850 \mathrm{~nm}$ ) is close to the peak value of roughly 86 percent, which is greater than SiPM sensors (5\%), and APD sensors (80\%), and provides for high sensitivity in sensing the light at these wavelengths.

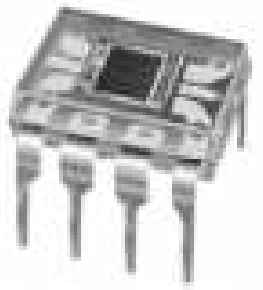

Fig.5 OPT101

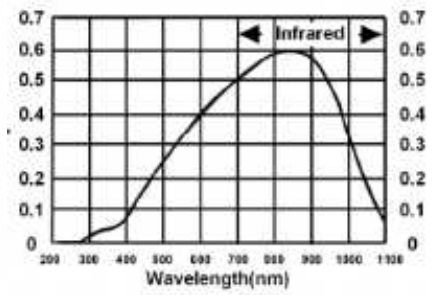

Fig.6 Special responsivity
The sensitivity of OPT101 does not stand out among other optoelectronic sensors described; yet, it is still good enough to sensitively assess hemodynamic fluctuations in the human body noninvasively. OPT101 also has a high gain and a reasonable cost. The OPT101 features a built-in transimpedance amplifier, which eliminates the need for additional electronics in clinical NIRS (Near Infrared Spectroscopy) instruments. It may also be readily incorporated with LEDs in a soft electrical circuit pad, making NIRS more portable and even wearables. The benefit of OPT101, which includes a sensitive-enough response. Structure with a small footprint. Its advanced electrical features and inexpensive cost make it ideal for recoding light intensity fluctuations at certain wavelengths to assess human body hemodynamics in our bedside care gadget design.

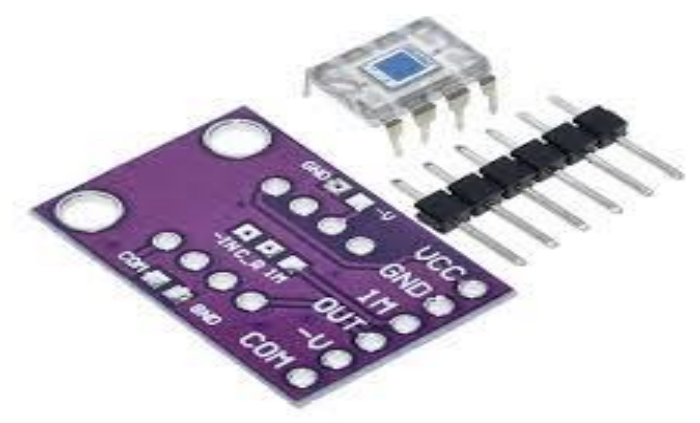


Fig.7 OPT101 with pins

OPT101 was used to gather light intensity data at a specific measuring point on the human body, and it then converted the optical readings to electrical signals, such as voltages signals. Our developed NIRS programme captured the output voltage response, converted it to physiological changes, and showed it. Calibrations for OPT101 independently or for each sensor array configuration is not needed by absolute measurements in our NIRS devices with OPT101. Due to the linear optoelectronics response and linear amplifier responses, the output voltage responses of the OPT101 increases linearly with lights intensity. Due to the fact that the optical density variation is computed by dividing the detected light intensity fluctuations by the initial detected light intensity baselines, which is a relative measurement, optical density variation is relative.

\section{IMPLEMENTATIONS}

PPG is achieved by reflecting or transmitting light that illuminate the relevant part of the body. An extension, like a finger, is connected to a lights source with a wavelength of so that when the light is transmitted across source straight to a photodetector it catches the light transmitted straight across the light source.

An example PPG signal consists of two components: a very huge DC component that flows through skin, muscles, and bone but does not pass-through blood vessels, a slight AC component that travels directly through blood vessels but does not pass-through skin, muscle, or bone, and a heartbeat signal resulting from light passing through arterial bloods vessels. After systole, the volume of bloods in arteries increases, while the light intensity received reductions. During diastole, the volume of blood in the arteries decreases, but light transmission increases. 99 percent of the signal in a basic measuring region originates from the skins, muscle, and bone, 0.9 percent from the vein, and 0.1 percent from the arteries.

PPG stands for photo electricals technique of measuring tissue blood volumes based on the changes in blood volume with each pulse. PPG has long been used to detect a variety of fundamental physiologic characteristics, including blood oxygen levels, heart rate, respiration, and blood pressures. PPG can also indicate other important cardiovascular (heart and blood vessel) characteristics, such as embolism. As a result, it's useful and vital to dig deep into the PPG signal in order to identify many physiologic characteristics with great precision.

The PPG signal is generated by the regular heartbeat. Because heart rate can be retrieved, it has a tight relationship to the beating duration. Another factor is that vascular flexibility and blood viscosity are affected by blood flow (resistance against fluidity). Furthermore, the PPG signal may be used to extract a wealth of cardiovascular (heart and blood vessel) data.

\section{A. Photoplethysmography $(P P G)$}

The region from $S$ to $M$ point in a typical PPG signal is the fast-beating phase, while the section from $\mathrm{M}$ to $\mathrm{P}$ point is the delayed phase. The PPG signal's major peak point is $\mathrm{M}$. The blood pressure (BP) is at its maximum point in the whole duration at this point. Another peak is the artiole's dicrotic wave, which is known as $\mathrm{Q}$ and demonstrates its adaptability. Dicrotic notch is the name given to the $\mathrm{P}$ point. The period between two primary peaks is used to calculate heart rate (tpp). The $\mathrm{M}-\mathrm{Q}$ time slots is a measure of the severity of arteriosclerosis (vessel stiffness). However, due to the existence of numerous circumstances, obtaining the features points for the genuine PPG signal is challenging.

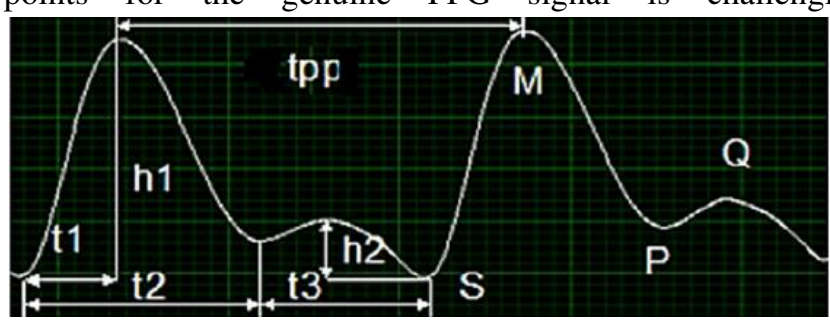

Fig.8 Standard PPG signal characteristics

PPG signals were acquired from the people's right index fingers as they sat in a relaxed position. Before the data was collected, the volunteers were given information about the study, their questions were answered, they were given explanations about Information about the data subjects and their privacy, and to consent and approve their participation in this study, they were given a consent form to fill out.

After receiving the $\mathrm{PPG}$ signals, several pre-processing processes were employed to remove the noise. To reduce noise in PPG data, a low-pass FIR filters with $\mathrm{N}=200$ points and a $10 \mathrm{~Hz}$ cut-off frequency is used as a pre-processing step. A 40-time domain feature extraction was performed on the PPG signal by utilizing the PPG signal's state after preprocessing, its first derivative after pre-processing, and its second derivative after pre-processing's. These characteristics are distributed as follows: 21 time-domains characteristics from the PPG signal, eight time-domain characteristics from the PPG signal's first derivative, seven time-domain characteristics from the PPG signal's second derivative, and four characteristics derived from the PPG signals first and second derivatives combined. The block diagram for extracting those features from the PPG signal as well as its derivatives is shown in Figure 9. 


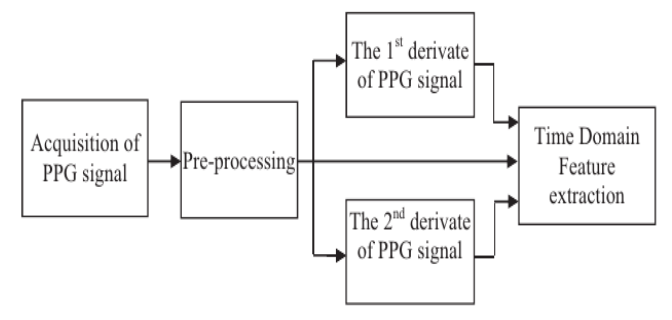

Fig.9 Block diagram PPG signal, and its derivatives

During the classification process, feature selections methods are frequently used to choose the important characteristics associated with the output labels while reducing the computational time. A primary goal of feature selection is to reduce classification costs by reducing the numbers of features, resulting in a more compact classifications model; a secondary goal is to enhance classification performances. In this study, we used RELIEF feature selection and correlationsbased feature selections as two different feature selection approaches.

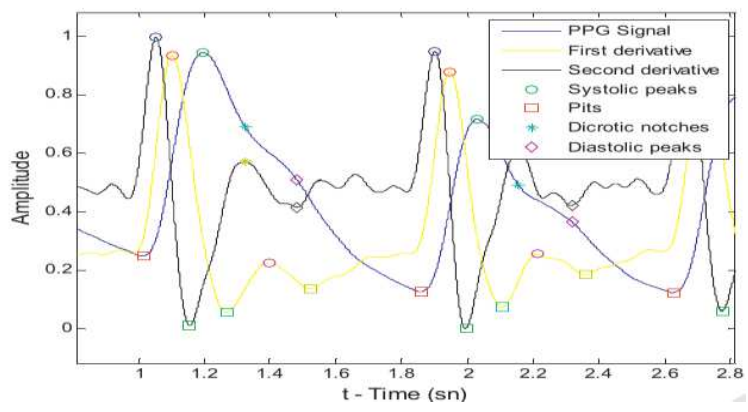

Fig.10 PPG signal, and the characteristic feature points

\section{1) RELEIF Feature Selection}

Selection of features based on RELIEF (RFS). RELIEF is a binary data classification feature selections technique proposed through Kira and Rendell in 1992. It has several powerful qualities since it is not dependent on the heuristic structure, and it just requires linear time used for the amount of provided features and trainings samples. It has a structure that works with both continuous and binary data. However, if there are superfluous characteristics and fewer training samples, it is unable to establish a meaningful differentiation. In their study, Kononenko et al improved a multiclass categorical dataset model with the RELIEFF method by using features such as generalization and strength for partial data. When PPG dataset with hemoglobin prediction was used to select features for RELIEFF-based feature selections, the number of features was reduced from 44 to 10 . TABLE 1

RELIEF feature selection

The RFS method was used to select the features (from 44 features to 10 features)

\begin{tabular}{|l|l|l|l|l|l|l|l|l|l|}
\hline 41 & 43 & 42 & 44 & 10 & 1 & 26 & 37 & 27 & 16 \\
\hline
\end{tabular}

2) Correlation Feature Selections

Feature selection based on correlation (CFS). Correlation is a criteria for determining whether or not a feature is related to other characteristics in the datasets and to outputs. Features are considered good if they add value to the class without being redundant with other relevant features. When using correlations, the favourable side of a characteristic is determined by whether or not it is significantly associated with the class or with any of the further features. To identify the important characteristics from all datasets, we utilised the correlation coefficients probability ( $\mathrm{p}$-value). The $\mathrm{p}$ values in this study were set to be $p<0.01$. The number of features was reduced from 44 to 11 when the CFS approach was applied to the PPG datasets with haemoglobin prediction. TABLE 2 CFS features selection

\begin{tabular}{|c|c|c|c|c|c|c|c|c|c|}
\hline \multicolumn{10}{|c|}{$\begin{array}{l}\text { The CFS method was used to select the features (from } 44 \text { features to } 11 \\
\text { features) }\end{array}$} \\
\hline 14 & 18 & 23 & 27 & 34 & 38 & 41 & 42 & 43 & 44 \\
\hline
\end{tabular}

\section{B. Separation Of Wavelength}

Shorter wavelengths of lights, such as blue and green, can only penetrate up to the capillary bed, whereas longer wavelengths, such as red and near infrared, may readily permeate farther over the skin and reach the arteries in the tissue. As demonstrated in Fig.10, There is considerable molecular extinction coefficient variation between the two species of hemoglobin among 650 and $1000 \mathrm{~nm}$. With low Attenuation levels at wavelengths between $600 \mathrm{~nm}$ and 950 $\mathrm{nm}$, a higher penetration level was observed.

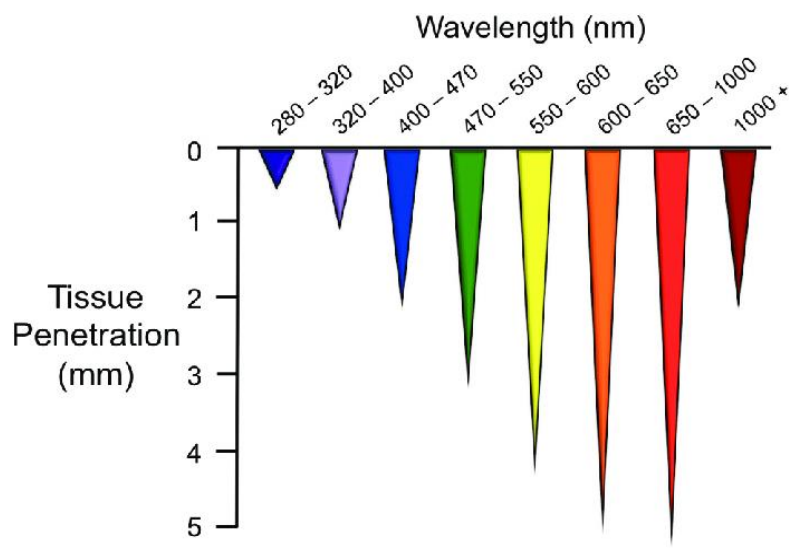

Fig.11 Different wavelengths penetration to skin

Deoxyhemoglobin blood is absorbed mainly at wavelengths between 600 and 800 nanometers, whereas oxyhemoglobin blood absorbs mostly at wavelengths between 820 and 1000 nanometers. Oxy-hemoglobin absorbs the same amount of light as deoxy-hemoglobin at $810 \mathrm{~nm}$, the isosbestic point.

Using wavelengths between 900 and $1000 \mathrm{~nm}$, we found substantial absorption for both oxygen and deoxyhemoglobin; the wavelength in our instance was $950 \mathrm{~nm}$. We have selected $670 \mathrm{~nm}$ as the second wavelength, which is the valley of oxyhemoglobins. Optical interactions between oxyhemoglobin and deoxy-hemoglobin occur at wavelength 810 $\mathrm{nm}$ (isosbestic point).

There was a noticeable absorption difference between oxyhemoglobin and deoxyhemoglobins at wavelengths $770 \mathrm{~nm}$ 
and $850 \mathrm{~nm}$ on each side of the interception. The wavelength selection may be altered, however, if high-quality LED sources are not readily available in this area.

\section{RESULTS AND DISCUSSIONS}

The results Hemoglobin and Blood Pressure are shown in the below figures. In those the plotted wave forms are absorbed and extracted using the PPG signal method. And these plots are classified as the oxy-hemoglobin, deoxy-hemoglobin, linear and kinetic of oxy-hemoglobin, saturation of oxyhemoglobin and deoxy-hemoglobin, ECG, Pulse, PPG $1^{\text {st }}$ derivative, PPG $2^{\text {nd }}$ derivative, Final systolic and diastolic.

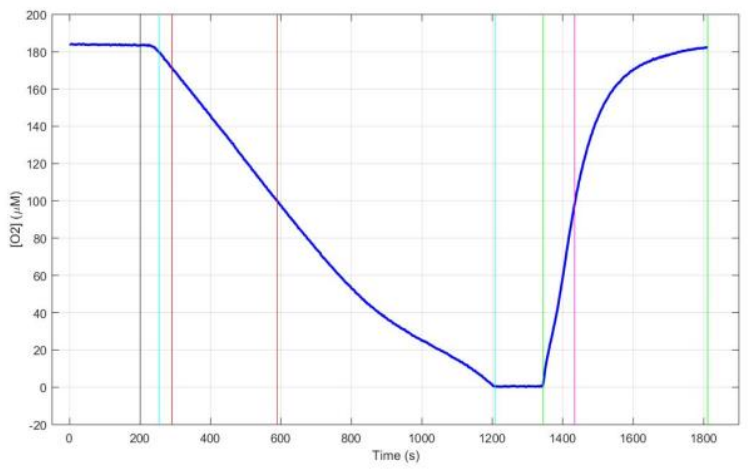

Fig.12 Oxy-Hemoglobin waveform

In the Oxy-hemoglobin waveform the wavelength is from $1200 \mathrm{~nm}$ to $1300 \mathrm{~nm}$ with respect to the oxygen (Micrometers) and time (seconds).

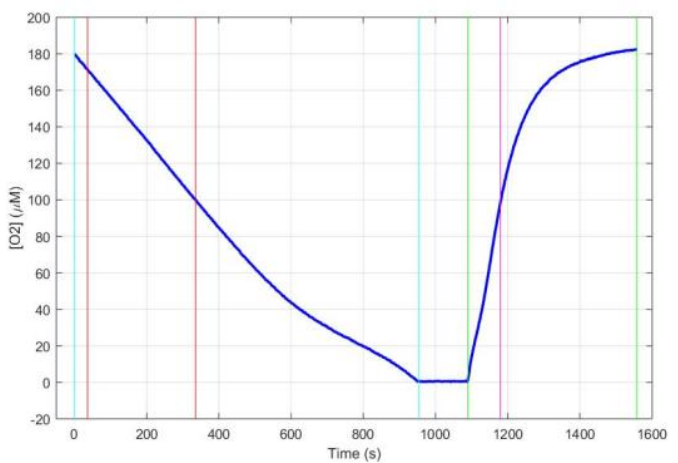

Fig.13 Deoxy-Hemoglobin waveform

In the deoxy Hemoglobin waveform, the wavelength is from $990 \mathrm{~nm}$ to $1150 \mathrm{~nm}$ with respect to Oxygen (micrometers) and Time (seconds).
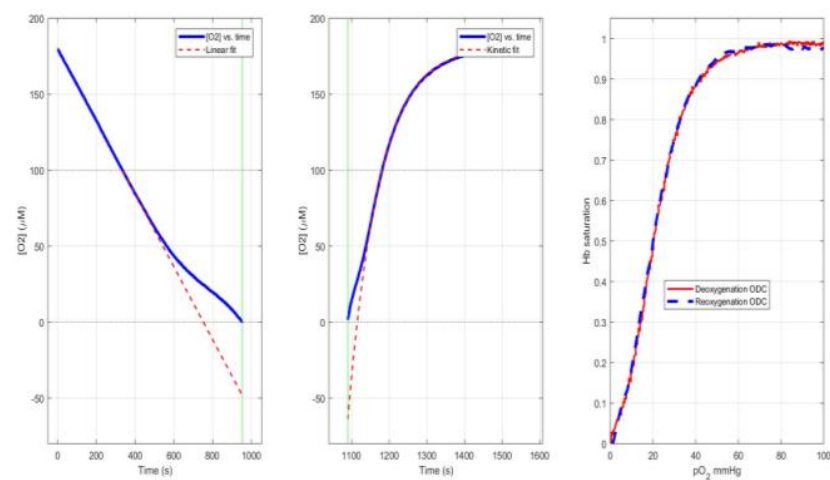

Fig.14 Linear and Kinetic fit of oxy-hemoglobin

In the Linear and Kinetic waveforms are formed by the combination of the both Deoxygenation and Reoxygenation.

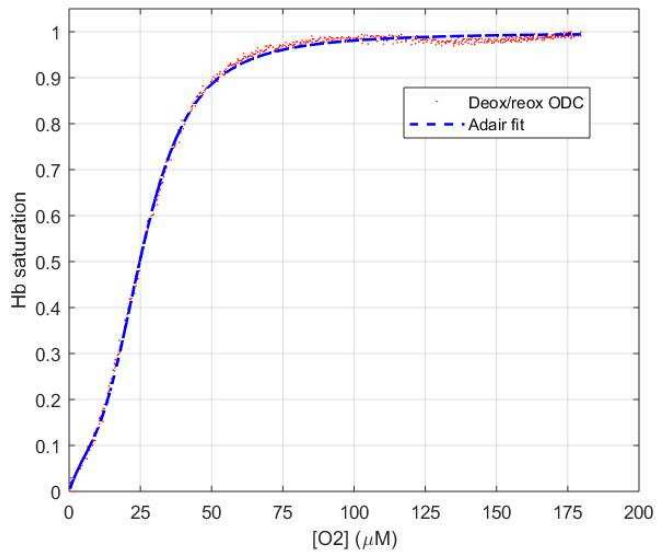

Fig.15 Saturation of Oxy-hemoglobin and Deoxy-hemoglobin's

In the above waveform the final output of the hemoglobin with combination of oxy-hemoglobin and deoxyhemoglobin's.

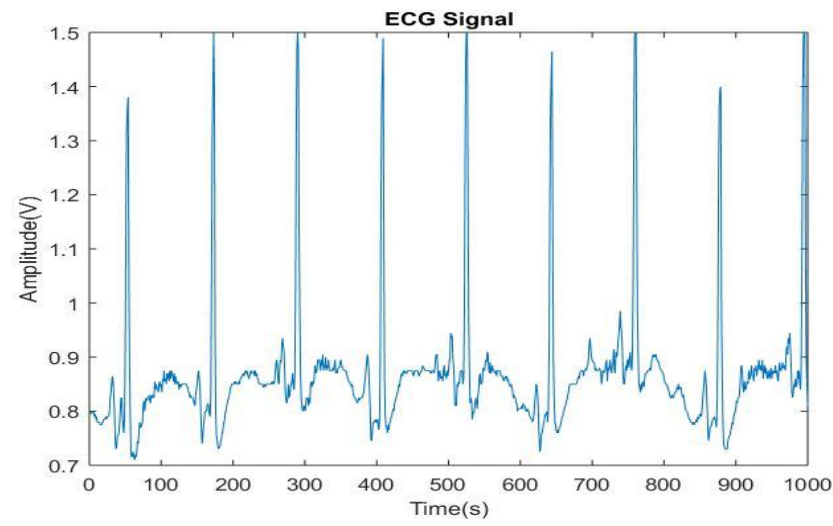

Fig.16 ECG signal

The above graph shows the ECG signal with respect to the Amplitude (Volts) and Time (Seconds). 


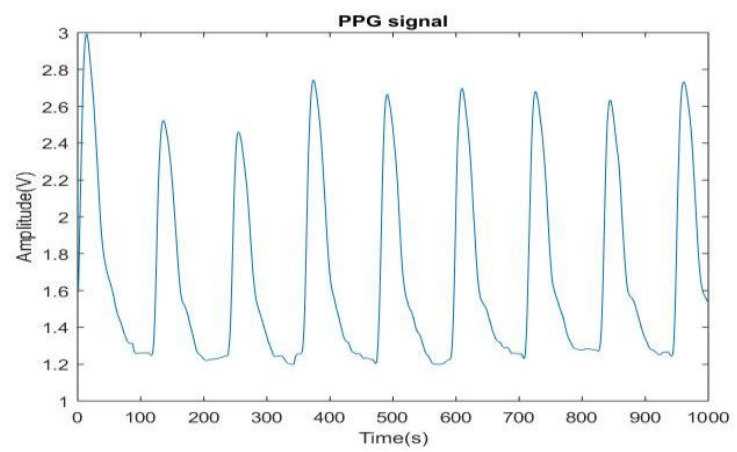

Fig.17 PPG signal

The above graph shows the PPG (Photoplethysmography) signal with respect to the Amplitude (Volts) and Time (Seconds).

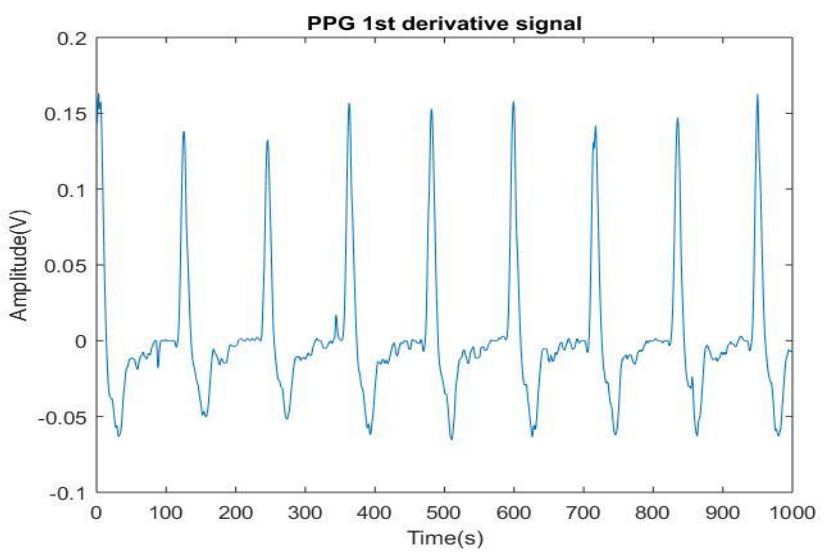

Fig.18 PPG signal $1^{\text {st }}$ Derivative

In the above graphs shows that the PPG signal $1^{\text {st }}$ Derivative with respect to the Amplitude (Volts) and Time (Seconds).

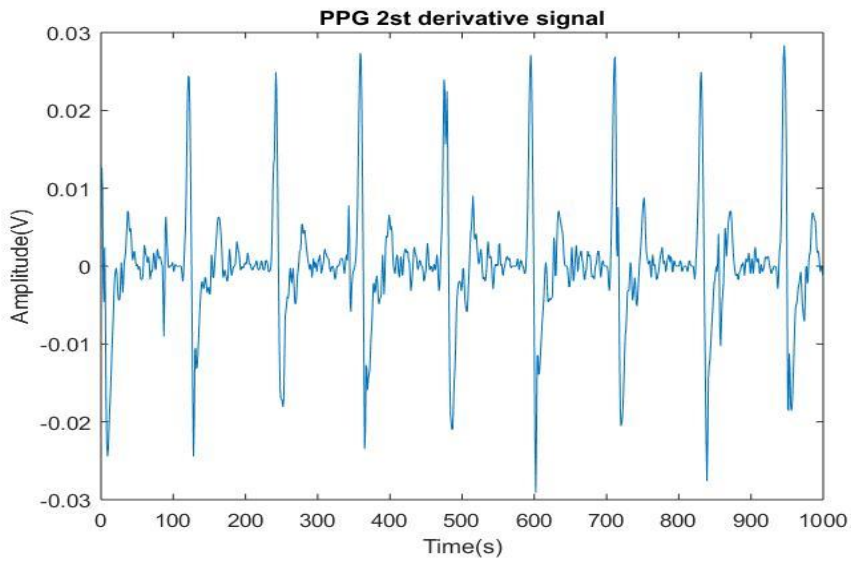

Fig.19 PPG signal $2^{\text {nd }}$ Derivative

In the above graphs shows that the PPG signal $2^{\text {nd }}$ Derivative with respect to the Amplitude(Volts) and Time (Seconds).

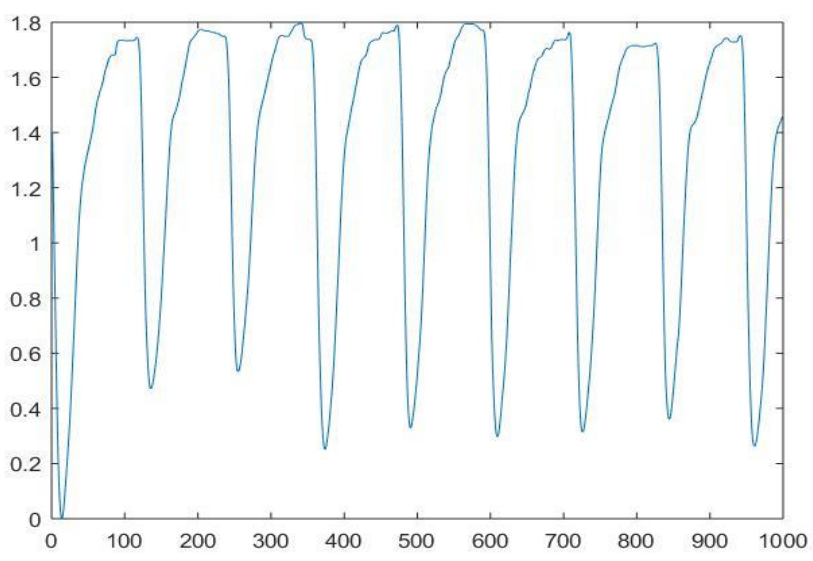

Fig.20 Pulse signal

The above graph shows the pulse signal with respect to the Amplitude (Volts) and Times (Seconds).
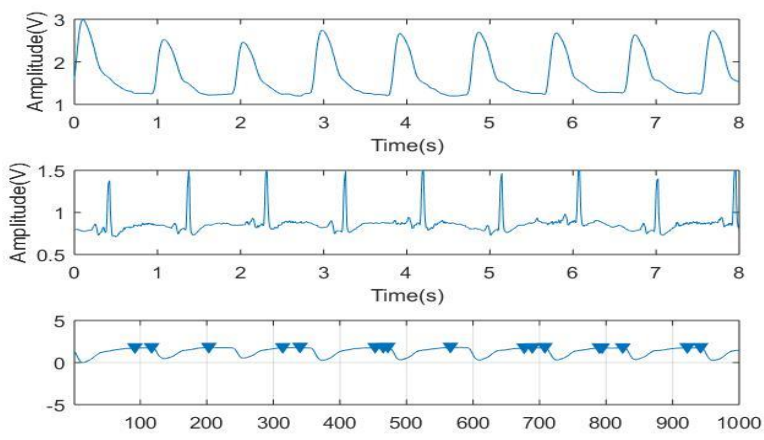

Fig.21 Final Systolic and Diastolic Signals

The above graph shows the combination of systolic and diastolic signals with respect to the Pulse and ECG signals.

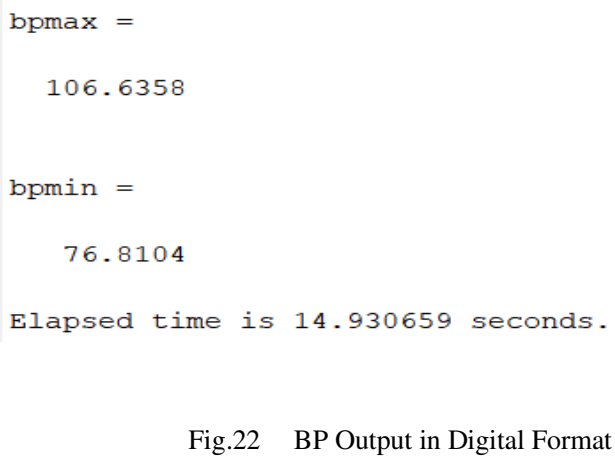

Fig.22 BP Output in Digital Format

he above figure shows the digital format output of the Blood Pressure with the respective time of $14.930659 \mathrm{~s} \cong 15 \mathrm{~s}$.

\section{CONCLUSION}

A non-invasive system based on multi wavelength light absorption for monitoring haemoglobin and blood pressure concentration has been disclosed. The newly designed sensor 
gadget can constantly monitor PPG signals at five different wavelengths. The sensor measurement will be validated based on additional measurements using an experimentals blood stream model, and sensor device will be optimised in the future.The wavelength for the Oxy-hemoglobin from the $820 \mathrm{~nm}$ to $1000 \mathrm{~nm}$ and the Deoxy-hemoglobin from the $600 \mathrm{~nm}$ to $800 \mathrm{~nm}$. The error rate between the Invasive and Non-Invasive method is $0.3598 \mathrm{dL}$.

In the future the error rate can decrease from $0.3598 \mathrm{dL}$ to $0.001 \mathrm{dL}$ by using the Non-Invasive method. These devices are portable and easy to carry anywhere and which works on the batteries. PPG signal design show good linearity in estimating the results with the regression coefficient. In conclusion, blood concentration and skin color affect the amount of PPG signal. This is pain free method rather than the invasive method and it is ecofriendly and cost efficient that everyone can afford it.

\section{REFERENCES}

[1] Serguei V.S. Pakhomov, Phd, James D. Buntrock, Ms, Christopher G. Chute.Automating the Assignment of Diagnosis Codes to Patient Encounters Using Examplebased and Machine Learning Techniques, J Am Med Inform Assoc. 2006 ; 13:516 -525.

[2] Gregor Lindner and Aristomenis K. Exadaktylos. How Noninvasive Haemoglobin Measurement with Pulse COOximetry Can Change Your Practice: An Expert Review. Department of Emergency Medicine, Inselspital, University Hospital Bern, Freiburgstrasse, 3010 Bern, Switzerland, 2013.

[3] Ramin Mohammadi1, Sarthak Jain, Stephen Agboola , Ramya Palacholla, Sagar Kamarthi, Byron C. Wallace. Learning to Identify Patients at Risk of Uncontrolled Hypertension Using Electronic Health Records Data. Northeastern University, Boston, MA, USA; 2 Partners Connected Health Innovation, Boston, MA, USA 3 Harvard Medical School, Boston, MA, USA.

[4] Han Chun Lin, Andrew Lowe, Ahmed M Al-Jumaily. Noninvasive blood pressure measurement algorithm using neural networks. Auckland University of Technology, Institute of Biomedical technologies, Auckland, New Zealand. May 22, 2014.

[5] J. Allen, Photoplethysmography and its application in clinical physiological measurement, Physiol. Meas. 28 (2007) R1-R39, March.

[6] Ball-Llovera A, Del Rey R, Ruso R, Ramos J, Batista O, Niubo I, editors. An Experience in Implementing the Oscillometric Algorithm for the Non-Invasive Determination of Human Blood Pressure. Proceedings of the Annual International Conference of the IEEE Engineering in Medicine and Biology Society; 2003.

[7] J.Kraitl, H. Ewald, U.Timm "Non-invasive measurement of blood components" IEEE fifth international Conference on Sensing Technology 2011.

[8] Suzaki, H.; Kobayashi, "Noninvasive measurement of total hemoglobin and hemoglobin derivatives using multiwavelength pulse spectrophotometry -In vitro study with a mock circulatory system" EMBS 28th Annual International Conference of the IEEE, 2006.
[9] Northrop RB. Noninvasive Instrumentation and Measurement in Medical Diagnosis. Boca Raton, Fla.: London: CRC ; Chapman \& Hall; 2001.

[10] Eoin OB, Gareth B, Gregory YHL. Blood pressure measurement. British Medical Journal. 2001; 322(7295): 1167-70. http://dx.doi.org/10.1136/bmj.322.7295.1167

[11] Association for the Advancement of Medical Instrumentation. American National Standard. Manual, electronic or automated sphygmomanometers. 2003; ANSI/AAMI SP10: 2002

[12] G.D. Jay, J. Racht, J. McMurdy, Z. Mathews, A. Hughes, S. Suner, G. Crawford, Point-of-care noninvasive hemoglobin determination using fiber optic reflectance spectroscopy, in: Proceedings of the 29th Annual International Conference of the IEEE, Engineering in Medicine and Biology Society. August 2007, 2007, pp. 2932-2935.

[13] S.H. Binns, D.D. Sisson, D.A. Buoscio, D.J. Schaeffer, Doppler ultrasonographic, oscillometricsphygmomanometric, and otoplethysmographic techniques for noninvasive bloodpressure measurement in anesthetized cats, J. Vet. Intern. Med. 9 (1995) 405-414, November-December. 